\title{
Effet de l'injection de somatotropine bovine recombinée (rbST) sur les performances de brebis laitières. Influence de la nature de l'aliment concentré *
}

\author{
V Dell'Orto 1, V Chiofalo 2, G Savoini 2, A Zumbo 2, \\ C Sgoifo-Rossi 1
}

1 Istituto di Alimentazione Animale, Facoltà di Medicina Veterinaria, Via Celoria 10, 20133 Milano; 2 Istituto di Zootecnica, Via S Cecilia 30, 98123 Messina, Italy

(Reçu le 12 janvier 1995; accepté le 3 janvier 1996)

\begin{abstract}
Résumé - Quarante brebis de race Comisana, produisant $0,970 \mathrm{~kg}$ de lait/jour ( $62 \pm 8$ jours de lactation), ont été nourries avec des aliments concentrés dont le rapport parois/amidon était variable. Quatre lots de 10 brebis chacun ont été constitués : i) HA : amidon haut 34,1\%; NDF 20,0\%; ii) HA + rbST ; iii) BA : amidon bas $12,2 \%$; NDF $32,7 \%$; iv) BA + rbST. Une injection de $320 \mathrm{mg}$ de somatotropine bovine recombinée (rbST) «retard" (Somidobove Ely Lilly Elanco) a été effectuée par voie souscutanée aux brebis des lots HA + rbST et BA + rbST. Le traitement par la rbST a augmenté de manière significative $(p<0,01)$ la production laitière, mesurée sur une période de 28 jours. Ni le traitement alimentaire ni la somatotropine n'ont modifié de manière significative les taux butyreux et protéique, tandis que le $\mathrm{pH}$ et le nombre de cellules somatiques du lait diminuaient de manière significative $(p<0,05)$ dans le lot HA. Aucune influence du traitement alimentaire et de la somatotropine n'a été mise en évidence sur l'aptitude à la coagulation du lait et sur les paramètres sanguins.
\end{abstract}

brebis / somatotropine / amidon / composition du lait

Summary - Effect of recombinant bovine somatotropin (rbST) administration on ewes milk. Influence of the nature of concentrate. Forty Comisana ewes (62 \pm 8 days in milk) were divided into four equal groups: i) HA: high starch 34.1\%; NDF 20.0\%; ii) HA + rbST; iii) BA: low starch $12.2 \%$, NDF $32.7 \%$ and iv) BA + rbST. Recombinant bovine somatotropin (Somidobove Ely Lilly Elanco) was injected once subcutaneously ( $320 \mathrm{mg} / \mathrm{ewe}$ ). Milk production was increased $(\mathrm{P}<0.01)$ by rbST treatment for a cycle of 28 days. High starch concentrate lowered significantly $(P<0.05)$ milk $p H$ and somatic cell counts. Dietary treatment and rbST did not affect, respectively, milk fat and protein percentages, rennet coagulation of milk and blood parameters.

\section{ewe / somatotropin / starch / milk composition}

* Recherche effectuée avec la participation financière du Conseil national des recherches de l'Italie, projet spécial RAISA, sous-projet N. 3. Publication N. 2231. 


\section{INTRODUCTION}

La composition du lait de brebis comme celui d'autres ruminants laitiers dépend de facteurs liés à l'animal (génétique, stade de lactation, âge, etc) et des caractéristiques du milieu, dont l'alimentation, qui influencent la production et la composition du lait (Hoden et al, 1985 ; Chiofalo et al, 1993). Des recherches ont clairement démontré que le rapport parois/amidon de la ration influence, chez la vache, les caractéristiques quantitatives et qualitatives du lait (taux butyreux et protéique) (Sauvant et al, 1994 ; Coulon et al, 1989). II est admis en effet que les aliments riches en parois se dégradent plus lentement dans le rumen que les céréales ; la digestion ruminale de l'amidon varie aussi largement en fonction de la nature de l'aliment et peut modifier la stabilité de l'écosystème ruminal (Robinson et al, 1986 ; Cerneau et Michalet-Doreau, 1991 ; Sauvant et al, 1994). Si la nature de l'aliment est l'un des facteurs de variation de la production et de la qualité du lait, le recours à la somatotropine bovine recombinée (rbST) peut être considérée comme une des techniques d'intensification de la production laitière (Cordonnier, 1989). L'influence de la rbST sur la production laitière a été largement démontrée et a fait l'objet depuis plusieurs années de très nombreux travaux (Peel et Bauman, 1987 ; Chilliard, 1988 ; Dell'Orto et Savoini, 1991 ; Bauman, 1992 ; Burton et al, 1994) chez la vache, mais peu d'études ont été faites chez la brebis. Stelwagen et al (1993) ont mis en évidence que la somatotropine augmente fortement et rapidement la production laitière, sans modifier les taux butyreux et protéique. Chez la vache, il a été montré que la bST d'une part et la composition des aliments concentrés d'autre part pouvaient modifier la production laitière. En revanche chez la brebis, on ne dispose pas de telles informations et il nous a semblé intéressant d'étudier simultanément les effets de ces deux facteurs, sur la production et la composition du lait, ainsi que sur son aptitude fromagère puisque c'est la destination principale du lait de brebis.

\section{MATÉRIEL ET MÉTHODES}

Quarante brebis de race Comisana (62 \pm 8 jours de lactation) produisant $0,970 \mathrm{~kg}$ de lait/jour, ont été réparties en quatre lots de dix brebis chacun : i) $\mathrm{HA}=$ concentré avec $34,1 \%$ d'amidon ; ii) $\mathrm{HA}+$ rbST ; iii) $B A=$ concentré avec $12,2 \%$ d'amidon ; iv) $B A+r b S T$.

La durée totale de l'essai a été de 40 jours (du 4 janvier au 15 février 1993) avec une période préexpérimentale de 12 jours et une période expérimentale de 28 jours.

Chaque jour, à 8 h 30 et à 19 h 00 , après la traite, les brebis reçevaient $0,6 \mathrm{~kg}$ brut de concentré (tableaux I et II), $0,5 \mathrm{~kg}$ brut de foin de vesce (MS 90,8\% ; MAT 7,9\%/MS ; NDF $65,3 \% / M S$ ) et pâturaient de 11 h 00 jusqu'à 16 h 00 une prajrie naturelle (MS $27 \%$; MAT 8,6\%/MS ; CB $26,1 \% / M S$; NDF $63 \% / M S)$. Le premier jour de la période expérimentale (jour 0 ), après la traite du matin, une dose de $320 \mathrm{mg} / \mathrm{brebis}$ de somatotropine bovine recombinée "retard" (Somidobove Ely Lilly Elanco) a été injectée par voie souscutanée aux brebis des lots HA + rbST et BA + rbST. Une fois par semaine pendant la période expérimentale, à la traite du soir $(16$ h 30$)$ et à celle du matin ( $6 \mathrm{~h} 00$ ), la production laitière individuelle a été contrôlée. Lors de ces contrôles, un échantillon par brebis de $250 \mathrm{~mL}$ de lait, représentatif des traites du soir et du matin a été constitué. Sur chacun de ces échantillons, des mesures de composition chimique et d'aptitude à la coagulation ont été réalisées. Les taux butyreux et protéique ont été déterminés à l'aide d'un «Milkoscan» (Foss Electric Hillerod Denmark). Le pH a été mesuré juste après la traite ( $\mathrm{pHmètre} \mathrm{Orion}$ mod EA 940). L'acidité titrable ( $\mathrm{SH} / 100 \mathrm{~mL})$ et la teneur en cellules somatiques du lait ont été déterminées à l'aide d'un «Fossomatic" (Foss Electric Hillerod Denmark). Les mesures rhéologiques ont été effectuées à l'aide d'un «Formagraph» (Foss Electric Hillerod Denmark), selon la méthode proposée par MacMahon et Brown (1982). Les paramètres rhéologiques mesurés ont été : le temps de coagulation ( $r$ ), le temps de raffermissement $\left(\mathrm{k}_{20}\right)$ et la fermeté du gel $\left(\mathrm{a}_{30}\right)$ en millimètre. Aux jours 0,7 et 21 un échantillon de 
Tableau I. Proportions (\%) des aliments entrant dans la composition des concentrés utilisés (HA : haute teneur en amidon et $\mathrm{BA}$ : basse teneur en amidon).

HA BA

$\begin{array}{lcl}\text { Pulpes de betterave déshydratées } & - & 36 \\ \text { Tourteau de soja (extraction) } & 18 & 19,5 \\ \text { Son fin de blé } & 9,5 & 11 \\ \text { Orge } & 16 & - \\ \text { Caroube } & 8 & 9 \\ \text { Maïs } & 23 & 8 \\ \text { Fève } & 5 & 5 \\ \text { Tourteau de tournesol (extraction) } & 4 & 4 \\ \text { Tourteau de lin } & 3 & 3 \\ \text { Remoulage de blé } & 9 & - \\ \text { Carbonate de calcium } & 2 & 2 \\ \text { Phosphate bicalcium } & 1 & 1 \\ \text { CMV a } & 1 & 1 \\ \text { Chlorure de sodium } & 0,5 & 0,5\end{array}$

a CMV x kg : Vit A 20000 UI ; Vit B2 4,4 mg; Vit B12 0,02 mg; Vit D3 $4000 \mathrm{UI}$; Vit E 4,5 mg; Vit PP $20 \mathrm{mg}$; Vit K $2,3 \mathrm{mg}$; acid d-panthothénique $10 \mathrm{mg}$; choline $220 \mathrm{mg}$; Co $0,30 \mathrm{mg}$; $\mathrm{Fe} 45 \mathrm{mg}$; I 3,2 mg; Mn 90 mg; Cu 6 mg; Zn 3,2 mg; BHT $10 \mathrm{mg}$.

Tableau II. Composition chimique des concentrés $(\% / \mathrm{MS})$.

\begin{tabular}{lrr} 
& $H A$ & $B A$ \\
\cline { 2 - 3 } & & \\
Matière sèche & 87,0 & 87,0 \\
Matières azotées totales & 20,0 & 19,9 \\
Cellulose brute & 6,7 & 14,9 \\
Constituants pariétaux NDF & 20,0 & 32,7 \\
Constituants pariétaux ADF & 8,7 & 16,7 \\
Amidon & 34,1 & 12,2 \\
Sucres & 5,8 & 5,7 \\
Lipides totaux & 1,4 & 1,9 \\
Cendres & 8,0 & 8,9 \\
UFL (kg/MS) & 1,07 & 0,88 \\
& &
\end{tabular}

sang a été prélevé à $8 \mathrm{~h} 00$, à jeun, pour chaque brebis dans un tube hépariné. Le sang a été immédiatement centrifugé et le plasma a été conservé à $-20{ }^{\circ} \mathrm{C}$ jusqu'au moment des analyses. La concentration des métabolites sanguins a été déterminée par différentes méthodes enzymatiques (Perkin Elmer $11 \mathrm{uv} / \mathrm{vis}$, longueur d'onde $546 \mathrm{~nm}$ ) : glucose (méthode GOD-PAP), cholestérol (méthode CHOD-PAP), triglycérides (méthode GPO-PAP) et protéines (méthode Biureto). L'analyse des facteurs (rbST) ef amidon et l'interaction amidon $x$ rbST ont été appliquées aux résultats des jours $7,14,21$ et 28 en prenant comme covariable la mesure de la même caractéristique au jour 0 (tableau III), avant l'administration de rbST et en utilisant le logiciel statistique SPSS/PC+.

\section{RÉSULTATS}

L'administration d'une seule dose de somatotropine à raison de $320 \mathrm{mg}$ par brebis a augmenté, sur 28 jours, significativement $(p<0,01)$ la production laitière (tableau IV). L'augmentation a été de $20,5 \%$ dans le lot $\mathrm{HA}+\mathrm{rbST}$ vs HA et de $35,8 \%$ dans le lot $\mathrm{BA}+\mathrm{rbST}$ vs BA. Par rapport au jour 0 (tableau III), l'effet exercé par la somatotropine a été plus marqué pour les brebis qui reçevaient le concentré à teneur plus élevée en amidon (+ 40,8\%). Après l'injection de rbST aux brebis, il y a eu une augmentation rapide et importante de la production laitière jusqu'au huitième jour environ (fig 1). Au septième jour en particulier le lot $\mathrm{HA}+$ rbST a eu une production plus élevée de $64 \%$ vs HA et de $17,5 \%$ vs BA + rbST, tandis que la production laitière des brebis du lot BA + rbST a été de $46,5 \%$ plus élevée vs $B A$. Aucune variation significative moyenne des taux butyreux et protéique du lait n'a été enregistrée (tableau IV), excepté dans le lot BA + rbST où il y a eu une augmentation non significative du taux butyreux de $13 \%$, le septième jour après le traitement avec la rbST (fig 2), tandis que le taux protéique du lait diminuait non significativement de $12 \%$ (fig 3 ), vs BA. Dans le même temps, la diminution du taux pro- 
Tableau III. Production laitière, composition et aptitude du lait à la coagulation : valeurs initiales brutes.

\begin{tabular}{lcccc} 
& $H A$ & & & \\
& & & $H A+r b S T$ & $B A+r b S T$ \\
\hline Production de lait (kg/jour) & 1,088 & 0,902 & 0,929 & 0,897 \\
Taux butyreux (\%) & 8,04 & 8,57 & 8,32 & 7,56 \\
Taux protéique (\%) & 5,96 & 5,83 & 5,94 & 5,60 \\
Matières grasses (g/jour) & 87,47 & 77,30 & 77,29 & 67,81 \\
Matières protéiques (g/jour) & 64,84 & 52,58 & 55,18 & 50,23 \\
pH & 6,67 & 6,74 & 6,74 & 6,8 \\
SH $/ 100 \mathrm{~mL}$ & 11,96 & 11,02 & 11,6 & 9,62 \\
Cell som $\times 10^{3} / \mathrm{mL}$ & 138 & 220 & 148 & 227 \\
r (minute) & 18,6 & 20,0 & 24,6 & 20,7 \\
$\mathrm{k}_{20}$ (minute) & 2,6 & 3,3 & 3,2 & 3,9 \\
$\mathrm{a}_{30}$ (mm) & 48,2 & 45,8 & 33 & 44,1 \\
& & & & \\
\hline
\end{tabular}

Tableau IV. Production laitière, composition et aptitude du lait à la coagulation : valeurs observées durant la période expérimentale, ajustées en tenant compte des différences initiales.

\begin{tabular}{lccccc}
\hline & $H A$ & $B A$ & $H A+r b S T$ & $B A+r b S T$ & $E S^{*}$ \\
\hline & & & & & \\
\hline & $1,085^{\mathrm{a}}$ & $0,883^{\mathrm{a}}$ & $1,308^{\mathrm{b}}$ & $1,199^{\mathrm{b}}$ & 81,3 \\
Production de lait (kg/jour) & 7,61 & 7,81 & 7,67 & 7,02 & 0,39 \\
Taux butyreux (\%) & 6,02 & 5,87 & 5,92 & 5,59 & 0,10 \\
Taux protéique (\%) & $82,6^{\mathrm{ac}}$ & $69,0^{\mathrm{a}}$ & $100,32^{\mathrm{b}}$ & $84,16^{\mathrm{bc}}$ & 8,20 \\
Matières grasses (g/jour) & $65,31^{\mathrm{a}}$ & $51,83^{\mathrm{b}}$ & $77,43^{\mathrm{c}}$ & $67,02^{\mathrm{ac}}$ & 6,40 \\
Matières protéiques (g/jour) & $6,57^{\mathrm{a}}$ & $6,64^{\mathrm{b}}$ & $6,59^{\mathrm{ab}}$ & $6,66^{\mathrm{b}}$ & 0,02 \\
$\mathrm{pH}$ & 12,11 & 11,36 & 11,98 & 10,13 & 0,43 \\
$\mathrm{SH} / 100 \mathrm{~mL}$ & $115^{\mathrm{a}}$ & $198^{\mathrm{b}}$ & $129^{\mathrm{ab}}$ & $140^{\mathrm{ab}}$ & 23 \\
Cell som x 103/mL & 15,9 & 18,8 & 18,2 & 18,1 & 1,9 \\
$\mathrm{r}$ (minute) & 2,7 & 2,9 & 2,9 & 3,0 & 0,3 \\
$\mathrm{k}_{20}$ (minute) & 53,3 & 48,5 & 47,2 & 49,1 & 5,40 \\
$\mathrm{a}_{30}$ (mm) & & & & & \\
& & $-1 . \cdots$ & & & \\
\hline
\end{tabular}

Les moyennes suivies de lettres différentes sont significativement différentes au seuil de $5 \%$. ${ }^{*}$ Erreur standard.

téique du lait du lot HA + rbST a été de 16,5 $\%$ vs $H A$. II faut souligner que la production de matières grasses (TB $\times$ PL) et de protéines (TP $\times$ PL) a augmenté chez les brebis traitées avec la rbST indépendamment de la nature du concentré distribué. Avec le concentré à teneur plus élevée en amidon il y a eu une baisse significative $(p<0,05) \mathrm{du}$ $\mathrm{pH}$ du lait $(-0,07)$ et du nombre de cellules somatiques $\left(-83 \times 10^{3} \mathrm{~mL}\right)$. L'aptitude à la coagulation du lait n'a pas été influencée significativement par la somatotropine et par le rapport parois/amidon du concentré. En revanche, il existe un effet du stade de lactation puisqu'on observe une diminution (tableau III) soit du temps de coagulation (r), soit du temps de raffermissement $\left(k_{20}\right)$ tandis que la fermeté du gel $\left(\mathrm{a}_{30}\right)$ augmente, exception faite pour le lot HA, où il n'y a pas eu de telles variations du $k_{20}$ (tableau IV). La nature du concentré et le traitement à la somatotropine n'ont pas modifié significati- 
Fig 1. Évolution de la production de lait lors de la période expérimentale.

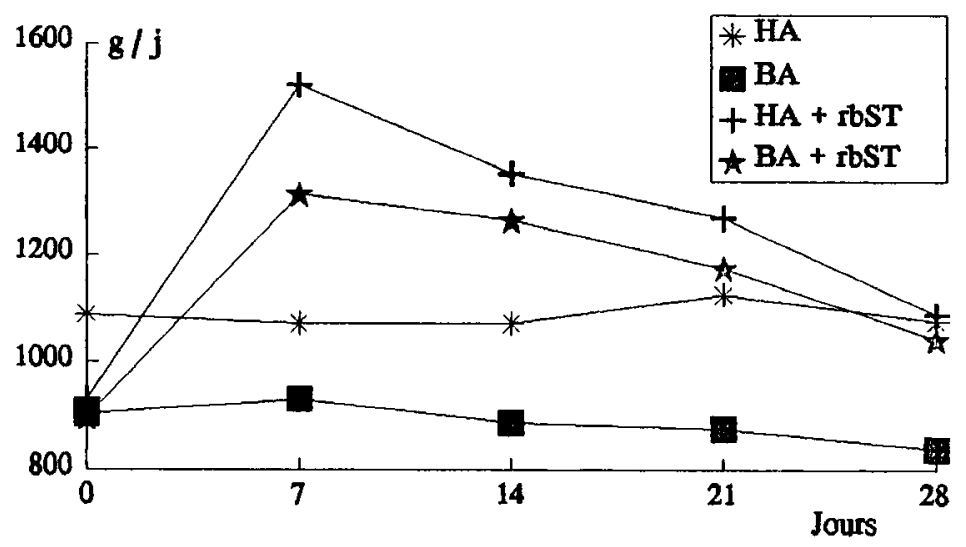

Fig 2. Évolution du taux butyreux du lait lors de la période expérimentale.
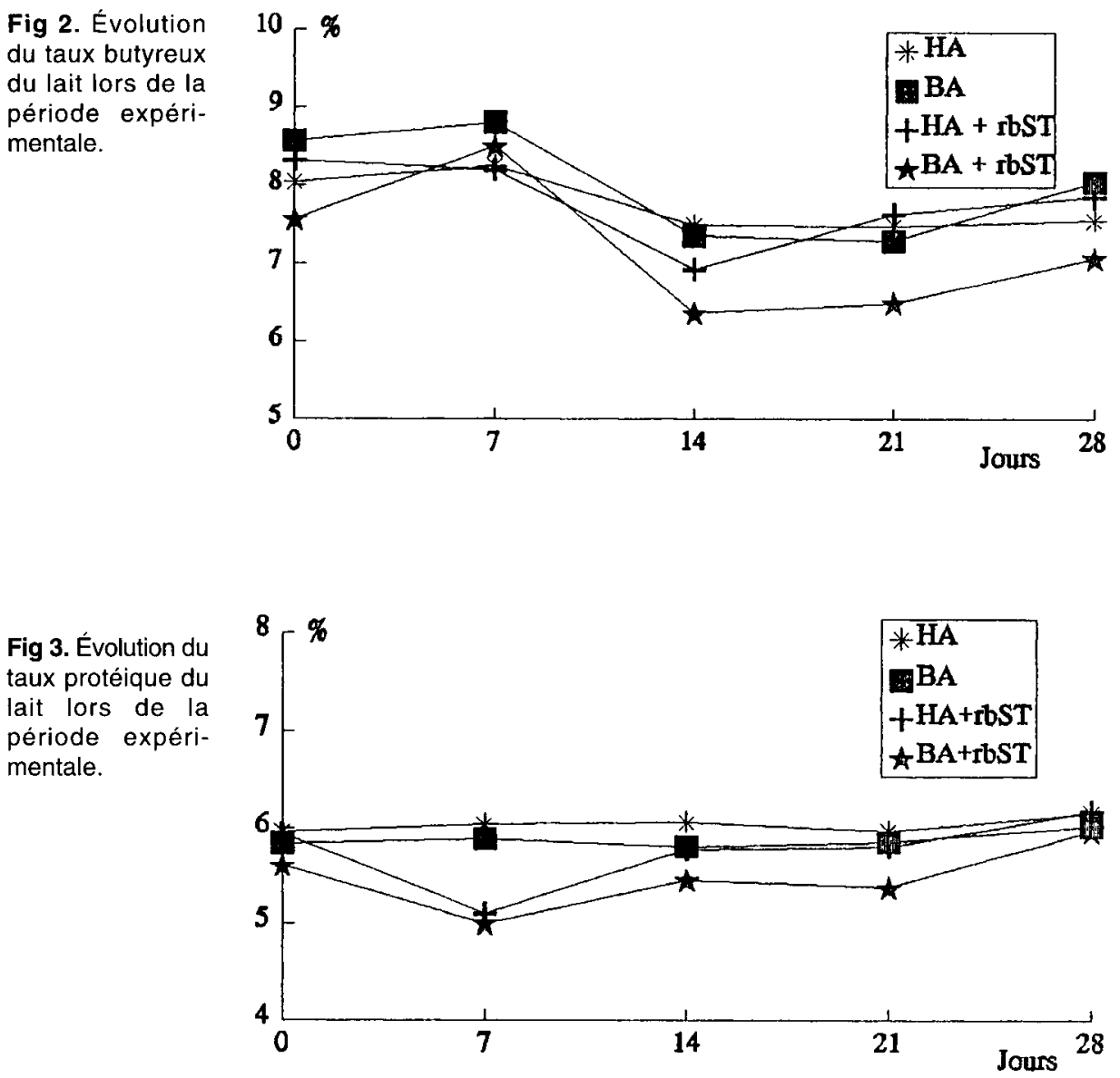
Tableau V. Paramètres métaboliques sanguins $(\mathrm{g} / 100 \mathrm{~mL})$.

\begin{tabular}{|c|c|c|c|c|c|c|c|c|c|c|}
\hline & & $H A$ & & $B A$ & & $H A+r b S$ & & $B A+r b S T$ & & $E S$ a \\
\hline$-\quad-$ & - & - & - & - & - & 一 & - & $-\quad-$ & - & $-\ldots$ \\
\hline Glucose & & 0,057 & & 0,056 & & 0,056 & & 0,055 & & 0,0012 \\
\hline Cholestérol & & 0,078 & & 0,075 & & 0,078 & & 0,077 & & 0,0019 \\
\hline Triglycérides & & 0,014 & & 0,014 & & 0,014 & & 0,014 & & 0,0007 \\
\hline Protéines & & 7,03 & & 6,86 & & 7,02 & & 6,97 & & 0,08 \\
\hline$\ldots$ & $\ldots$ & $\ldots$ & - & - & $\ldots$ & - & - & - & - & - \\
\hline
\end{tabular}

vement les teneurs du sérum en glucose, cholestérol, triglycérides et protéines (tableau V).

\section{DISCUSSION}

Cette étude a donc confirmé l'effet galactopoiétique exercé par la somatotropine, en augmentant fortement la production laitière des brebis. La distribution de concentrés ayant des proportions variables en parois et amidon aux brebis traitées par la rbST a donné des réponses différentes. Ainsi, immédiatement après le traitement par la rbST, la disponibilité plus élevée en énergie pour les brebis du lot HA + rbST a permis d'élever plus fortement et plus rapidement la production de lait. Avec le concentré plus riche en parois facilement digestibles (pulpes de betteraves), mais moins riche en énergie, la production de lait a été moins augmentée (fig 1). Conformément aux résultats obtenus par Stelwagen et al (1993), la somatotropine n'a pas provoqué de changement de composition du lait dans cet essai, probablement parce que les brebis sont restées en moyenne en bilan énergétique positif, si l'on excepte probablement un bilan énergétique négatif à court terme ( $48 / 72$ heures) après l'injection de somatotropine chez les brebis alimentées avec le concentré riche en parois, ce qui peut expliquer l'augmentation du taux butyreux observée au septième jour.
En effet, un accroissement de la production de lait et un bilan énergétique et azoté négatif, avec une chute du taux protéique du lait ont été mis en évidence chez les vaches laitières (Chilliard, 1988). L'augmentation de la production de matières protéiques a été trouvée aussi par Stelwagen et al (1993). À moyen terme, on observe également une diminution du taux protéique du lait des brebis traitées à la bST (fig 3), ce qui est conforme aux évolutions observées chez les ruminants en bilan énergétique négatif. Toutefois, l'accroissement de la production laitière sous l'effet de la bST permet une augmentation des quantités de protéines exportées dans le lait comme cela a déjà été observé chez les vaches (Chilliard, 1988 ; Vérité et al, 1988). La fermentescibilité élevée de l'amidon du concentré distribué au lot HA a déterminé la baisse du $\mathrm{pH}$ et du nombre de cellules somatiques du lait. En fait, cette diminution des cellules est très dépendantes de l'état sanitaire des mamelles et peut beaucoup varier entre brebis. La somatotropine n'a pas modifié les caractéristiques rhéologiques du lait. Laurent et al (1992) ont confirmé l'absence d'effet sur les paramètres de la coagulation du lait chez les vaches laitières dont ils ont examiné particulièrement la composition des protéines du lait afin d'évaluer un effet sur les caséines du lait, mais aussi sur la taille des micelles, sans observer de variations significatives. Dans notre essai, par rapport au jour 0 , il y a eu amélioration 
des caractéristiques de coagulation du lait, par diminution du temps de coagulation, du temps de raffermissement et augmentation de la fermeté du gel, ce qui correspond à un accroissement de l'aptitude fromagère du lait avec le stade de lactation. Aucune variation significative des paramètres sanguins n'a été observée. S'il y a eu des modifications dans les métabolites sanguins, elles ont été, probablement, limitées à la période immédiatement après le traitement avec la rbST, pendant laquelle l'administration de somatotropine a déterminé un bilan énergétique et azoté négatif.

\section{CONCLUSION}

Les données obtenues confirment que la somatotropine bovine recombinée, administrée à des brebis laitières, augmente la production sans modifier significativement la composition chimique du lait. L'utilisation de concentrés ayant différentes teneurs en parois et en amidon n'a pas entraîné de variations significatives de la qualité du lait destiné à la fabrication fromagère. II reste à établir quelle est la dose idéale de somatotropine à injecter aux brebis en fonction du niveau alimentaire choisi, compte tenu d'une situation favorable à la production du lait destiné à la transformation sans problème de quotas.

\section{RÉFÉRENCES}

Bauman DE (1992) Bovine somatotropin: review of an emerging animal technology. J Dairy Sci 75, 34323451

Burton JL, Mc Bride BW, Block E, Glimm DR, Kennelly JJ (1994) A review of bovine growth hormone. Can J Anim Sci 74, 167-201
Cerneau P, Michalet-Doreau B (1991) In situ starch degradation of different feeds in the rumen. Reprod Nutr Dev 31, 65-72

Chilliard Y (1988) Rỏles et mécanismes d'action de la somatotropine (hormone de la croissance) chez les ruminants en lactation. Reprod Nutr Dev 28, 39-59

Chiofalo V, Savoini G, Micari P, Zumbo A, Bontempo $V$, Ziino M (1993) Impiego di differenti fonti energetiche per l'alimentazione quanti-qualitative del latte. Atti $X^{\circ}$ Congr Naz Ass Sc Prod Anim 339-344

Cordonnier $\mathrm{P}$ (1989) Impact économique de l'utilisation de la somatotropine dans les systèmes laitiers français. INRA Prod Anim 2, 313-316

Coulon JB, Faverdin P, Laurent F, Cotto G (1989) Influence de la nature de l'aliment concentré sur les performances des vaches laitières. INRA Prod Anim $2,47-53$

Dell'Orto V, Savoini G (1991) Recombinant bovine somatotropin (rbST) treatment in dairy cows: effects on ruminal activity and milk properties. Microb Alim Nutr 9. 121-132

Hoden A, Coulon JB, Dulphy JP (1985) Influence de l'alimentation sur la composition du lait. Effets des régimes alimentaires sur les taux butyreux et protéique. Bull Tech CRZV Theix INRA 62, 69-79

Laurent F, Vignon B, Coomans D, Wilkinson J, Bonnel A (1992) Influence of bovine somatotropin on the composition and manufacturing properties of milk. J Dairy Sci 75, 2226-2234

MacMahon DJ, Brown RJ (1982) Evaluation of Formagraph for comparing rennet solutions. J Dairy $S c i$ 65, 1639-1642

Peel CJ, Bauman DE (1987) Somatotropin and lactation. J Dairy Sci 70, 474-486

Robinson PH, Tamminga S, Van Vuuren AM (1986) Influence of declining level of feed intake and varying the proportion of starch in the concentrate on rumen fermentation in dairy cows. Livest Prod Sci 15, 175-189

Sauvant $D$, Chapoutot $P$, Archimède $H$ (1994) La digestion des amidons par les ruminants et ses conséquences. INRA Prod Anim 7, 115-124

Stelwagen K, Grieve DG, Walton JS, Ball JL, McBride BW (1993) Effect of prepartum bovine somatotropin in primigravid ewes on mammogenesis, milk production, and hormone concentrations. J Dairy Sci 76, 992-1001

Vérité R, Rulquin H, Faverdin P (1988) Effect of slow released somatotropin on dairy cow performances. In: Use of Somatotropin in Livestock Production (K Sejrsen, M Vestergaard, A Neimann-Sorensen, eds), Elsevier Appl Sci Publ, Londres, 269 